\title{
Review of: "A single-cell transcriptomic landscape of innate and adaptive intratumoral immunity in triple negative breast cancer during chemo- and immunotherapies"
}

hecheng li

Potential competing interests: The author(s) declared that no potential competing interests exist.

The authors assessed the immune landscape in the tumour microenvironment of two TNBC mouse models using SCRNA-seq technology. Immune cells were evaluated in untreated conditions and after being treated with chemotherapy and/or immunotherapy. The immune TME in this in vivo study can lead to new insights on the immune characteristics of TNBC and researchers will strive to discover the immune landscape of TNBC in patients. While there are some limitations for this study.

1.These two cell lines have very different biological characteristics with regard to proliferation and metastasis. The authors should conduct signaling pathways analysis related to cell proliferation and migration in these two cell lines. Such assessment might help to further understand the role of immune status in the molecular biology of tumor cells.

2. In the subcluster analysis, the authors classfied subcluers based on gene expressions. It is better to validate the gene expression by classical methods. Use the tumor samples to prepare single-cell suspensions and flow cytometry to identify the sub cluster cells by specific markers. Perform qRT-PCR to validate the difference of gene expression levels. 